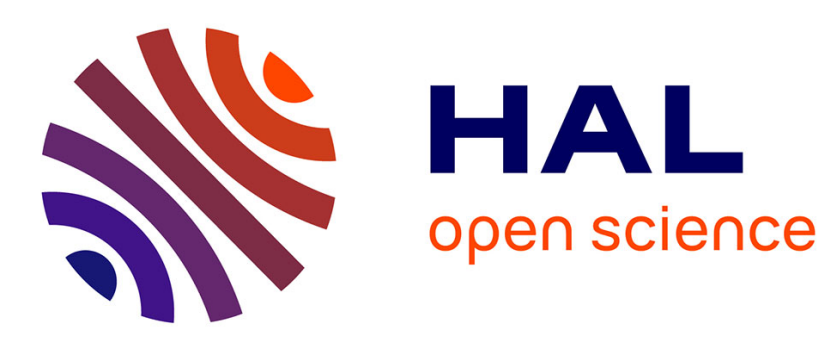

\title{
Numerization of a memory effect for an homogenized composite material with a large contrast in the phase thermal conductivities
}

\author{
David Dureisseix, Pascale Royer, Béatrice Faverjon
}

\section{To cite this version:}

David Dureisseix, Pascale Royer, Béatrice Faverjon. Numerization of a memory effect for an homogenized composite material with a large contrast in the phase thermal conductivities. International Journal of Heat and Mass Transfer, 2015, 90, pp.140-148. 10.1016/j.ijheatmasstransfer.2015.06.048 . hal-01171181

\author{
HAL Id: hal-01171181 \\ https://hal.science/hal-01171181
}

Submitted on 1 Nov 2016

HAL is a multi-disciplinary open access archive for the deposit and dissemination of scientific research documents, whether they are published or not. The documents may come from teaching and research institutions in France or abroad, or from public or private research centers.
L'archive ouverte pluridisciplinaire HAL, est destinée au dépôt et à la diffusion de documents scientifiques de niveau recherche, publiés ou non, émanant des établissements d'enseignement et de recherche français ou étrangers, des laboratoires publics ou privés. 


\title{
Numerization of a memory effect for an homogenized composite material with a large contrast in the phase thermal conductivities*
}

\author{
David Dureisseix ${ }^{1, \dagger}$, Pascale Royer ${ }^{2}$ and Béatrice Faverjon ${ }^{1,3}$
}

1 Université de Lyon, INSA-Lyon, LaMCoS, CNRS UMR 5259, 18-20 Rue des Sciences, bâtiment d'Alembert, F-69621 Villeurbanne, France

2 Laboratoire de Mécanique et Génie Civil, LMGC, Université Montpellier, CNRS, CC048, Place E. Bataillon, F-34095 Montpellier, France

3 School of Mechanical and Manufacturing Engineering, UNSW Australia, Sydney, NSW 2052, Australia

$\dagger$ Corresponding author

\begin{abstract}
In this article, we propose a systematic numerical solution method for deriving the homogenized material parameters in the case where a large contrast in the phase thermal properties leads to a macroscopic memory effect. Focus is therefore set on the determination of this memory effect for a periodic microstructure. As for other and more classical homogenized parameters, the possibility of analyzing with the finite element method a single periodic cell is used, and a transient simulation allows to provide the incremental evolution of the memory effect function. Additionally, some approximations are proposed for a low cost estimate of this function, and validated on two examples.

This is a preprint of the following article: David Dureisseix, Pascale Royer and Béatrice Faverjon, Numerization of a memory effect for an homogenized composite material with a large contrast in the phase thermal conductivities, International Journal of Heat and Mass Transfer 90:140-148, (C) 2015, Elsevier, DOI: 10.1016/j.ijheatmasstransfer.2015.06.048. Licensed under the Creative Commons CCBY-NC-ND 4.0 http://creativecommons.org/licenses/by-nc-nd/4.0/
\end{abstract}

Keywords: computational material; asymptotic expansion; 2-field model; periodic media; thermal conduction problems

PACS: $44.05 .+\mathrm{e} ; 44.10 .+; 44.35 .+\mathrm{c}$

MSC: $35 \mathrm{~B} 27 ; 35 \mathrm{~K} 05$

\section{Introduction}

Models with memory effects (or heredity models) have been used quite extensively in structural mechanics for viscoelasticity behaviors. Especially when the structures are subjected to forced vibration loading, their response may be easily found, analytically in simple cases, or numerically. For transient responses, concurrent models, mainly with internal variables have proved their ability to be used numerically [1].

Several justifications can be stated for the physical meaning of memory effects, one being the presence of a microstructure in the material, leading to a delayed response at the macroscopic scale, therefore related to upscaling [2] and a kind of time non-locality. This is for instance the case for scattering of elastic waves for hyperbolic problems [3], for composite materials where the constituents themselves exhibit a memory effect [4], or for particular non-linear behaviors or time-dependent material properties $[5]$.

In this article we focus on the case of evolution (elliptic) problems, even with linear constituents, but where the contrast of constitutive properties is large. The delayed effect is therefore directly provided by the homogenization procedure $[6,7,8,9]$. We proposed to study a thermal problem for illustration purpose, though the same framework also applies to different physics [10].

*D. Dureisseix gratefully acknowledges support and partial funding from Carnot Institute 'Ingénierie@Lyon' (Carnot $\mathrm{I} @ \mathrm{~L})$, and B. Faverjon gratefully acknowledges the French Education Ministry, University of Lyon, CNRS, INSA of Lyon and LabEx iMUST for the CRCT and the out mobility grant. 
The aim is to be able to compute numerically the memory effect function as a macroscopic material characteristic from simulations on the microstructure (i.e. a periodic cell), as it is classically done for the homogenized other parameters, such as the capacity and the conductivity.

In this article, section 2 recalls the results of the periodic homogenization method for the kind of problem that is under concern. Section 3 proposes a numerical procedure for the memory effect function determination. Finally, section 4 provides two numerical examples: (i) a 1D problem with an analytical solution, allowing to test the numerical procedure, and whose study provides approximations for determining the memory function at small and large times; (ii) a 3D problem for testing the discretization choices and for validation of the previous approximations.

\section{Asymptotic expansion and periodic homogenization}

This homogenization technique is based on the spatial description of the various fields at two scales, i.e. with two coordinate systems: one is the slow coordinates $x$ at the scale of the whole structure (typically $x \in[0, L]$ where $L$ is a characteristic size of the whole structure), the other one is the fast coordinate $y$, related to the scaled microstructure cell (typically $y \in[0, l / \varepsilon]$ where $l$ is the characteristic size of the cell). The scaling parameter is $\varepsilon=l / L$; it is expected to be small, traducing the assumption that the scales are separated. The spatial derivative then reads $\frac{\partial}{\partial x}+\frac{1}{\varepsilon} \frac{\partial}{\partial y}$. The temperature field is expanded with respect to the (small) parameter $\varepsilon$ :

$$
\theta=\sum_{\alpha=0}^{\infty} \varepsilon^{\alpha} \theta_{\alpha}(x, y, t)
$$

where all the temperature fields $\theta_{\alpha}(x, y, t)$ are $\Omega$-periodic for the variable $y$, where $\Omega$ is the scaled basic cell that allows the reconstruction of the whole microstructure by periodic duplication in each space dimension. For sake of simplicity, we assume that this microstructure is composed of two phases, denoted by $F$ and $S$ occupying the domains $\Omega_{F}$ and $\Omega_{S} ; \Gamma=\partial \Omega_{S} \cap \partial \Omega_{F}$ is the interface. Equating all the problem equations for the different powers of $\varepsilon$ allows to successively provide the models for the different temperature fields $[11,12]$.

The large contrast in material characteristics arises when the ratio between the capacities or the conductivities is of the order of $\varepsilon^{n}$ with $n \neq 0$. A special case arises when the ratios between conductivity is of order $\varepsilon^{2}$ while the capacities are of the same order, i.e. $\rho_{F} c_{F} \approx \rho_{S} c_{S}$ and $k_{F}=\varepsilon^{2} k_{F}^{\prime}$ with $k_{F}^{\prime} \approx k_{S}$. In such a case, the emergent macroscopic thermal problem is of a different kind [7]. This case is usefully interpreted with characteristic times $[9,13]$ since the characteristic macroscopic time is expected to be $\tau_{M}=\left(C_{M} / k_{M}\right)(L / \pi)^{2}$ (if $C_{M}$ and $k_{M}$ are the homogenized total capacity and conductivity), while their counterparts for the microscopic $S$ and $F$ phases are $\tau_{S}=\left(\rho_{S} c_{S} / k_{S}\right)\left(l_{S} / \pi\right)^{2}$ and

$$
\tau_{F}=\frac{\rho_{F} c_{F} l_{F}^{2}}{k_{F} \pi^{2}} \approx \frac{\rho_{F} c_{F} L_{F}^{2}}{k_{F}^{\prime} \pi^{2}} \approx \tau_{S} / \varepsilon^{2} \approx \tau_{M}
$$

since the characteristic lengths associated to each phase are $l_{S} \approx l_{F} \approx l$ and $L_{S}=l_{S} / \epsilon \approx L_{F}=l_{F} / \epsilon \approx L$. Therefore, the transient phenomena at the micro scale for the $F$-phase may well arise at macro scale.

Details on the successive derivations of the micro and macro problems can be found in [7], and the main results are recalled in the following.

\subsection{Macroscopic property of $\theta_{S 0}$}

The first result for the $S$-phase is that the temperature at order 0 , i.e. multiplied by $\varepsilon^{0}$, does not depend on the fast variable:

$$
\theta_{S 0}=\theta_{S 0}(x, t)
$$

it is therefore a macroscopic temperature field, and its gradient with respect to the fast variable $y$ is null. Its gradient with respect to the slow variable $x$ is a macroscopic temperature gradient

$$
\underline{Z}_{S 0}(x, t)=\underline{\operatorname{grad}}_{x} \theta_{S 0}
$$




\subsection{Localization property of $\theta_{S 1}$}

The next order equations give the problem that $\theta_{S 1}$ should satisfy:

$$
\begin{array}{r}
\operatorname{div}_{y} \underline{q}_{S 0}=0 \quad \text { in } \Omega_{S} \\
\underline{q}_{S 0} \cdot \underline{n}_{S}=0 \text { on } \Gamma \\
\underline{q}_{S 0}=-k_{S}\left(\underline{Z}_{S 0}+\underline{\operatorname{grad}}_{y} \theta_{S 1}\right) \text { in } \Omega_{S} \\
\theta_{S 1} \text { is } \Omega \text {-periodic }
\end{array}
$$

It is a steady-state thermal problem whose variational formulation is

$$
\int_{\Omega_{S}} \underline{\operatorname{grad}}_{y} \theta^{\star} \cdot k_{S} \underline{\operatorname{grad}}_{y} \theta_{S 1} d \Omega=-\int_{\Omega_{S}} \underline{\operatorname{grad}}_{y} \theta^{\star} \cdot k_{S} \underline{Z}_{S 0} d \Omega
$$

for all $\Omega$-periodic test functions $\theta^{\star}(y)$. It is usually discretized with finite elements, and its solution is linear with respect to the macroscopic loading $\underline{Z}_{S 0}$. Therefore this solution, and its gradient $\underline{Z}_{S 1}=$ $\operatorname{grad}_{y} \theta_{S 1}$ can be expressed using a linear operator which is a characteristic of the $S$-microstructure. For instance,

$$
\underline{Z}_{S 1}(x, y, t)=-\boldsymbol{L}_{S}(y) \underline{Z}_{S 0}(x, t)
$$

The numerical determination of $\boldsymbol{L}_{S}$ requires: (i) as many resolutions of the previous linear micro problem (1) on $\Omega_{S}$ as there are independant components in $\underline{Z}_{S 0}$, i.e. 2 for $2 \mathrm{D}$ problems, and 3 for 3D problems, and (ii) the storage of the same number of temperature gradient fields on $\Omega_{S}$ (the columns of the matrix $\boldsymbol{L}_{S}$ ).

This operator is a localization operator for the temperature gradient. Indeed, it allows to recover the first two terms of the full temperature gradient, obtained from the asymptotic expansion of $\theta_{S}$, once the macroscopic gradient is known:

$$
\underline{Z}_{S} \approx \underline{Z}_{S 0}(x, t)+\underline{Z}_{S 1}(x, y, t)=\left(\mathbf{1}-\boldsymbol{L}_{S}\right) \underline{Z}_{S 0}
$$

and of the thermal flux

$$
\underline{q}_{S}=-k_{S} \underline{Z}_{S} \approx \underline{q}_{S 0}=-k_{S}\left(\mathbf{1}-\boldsymbol{L}_{S}\right) \underline{Z}_{S 0}
$$

\subsection{Problem to solve for $\theta_{F 0}$}

The zero-order terms for the $F$-phase provide the following problem:

$$
\begin{array}{r}
\rho_{F} c_{F} \dot{\theta}_{F 0}=-\operatorname{div}_{y} \underline{q}_{F 1} \text { in } \Omega_{F} \\
\theta_{F 0}=\theta_{S 0} \quad \text { on } \Gamma \\
\underline{q}_{F 1}=-k_{F}^{\prime} \underline{\operatorname{grad}}_{y} \theta_{F 0} \text { in } \Omega_{F} \\
\theta_{F 0} \quad \text { is } \Omega \text {-periodic }
\end{array}
$$

This problem couples the micro and the macro scales due to the fact that $\theta_{F 0}$ still depends on the $y$ coordinate, and due to the presence of the $\theta_{S 0}(x, t)$ term as a boundary condition on $\Gamma$. On the $F$-cell, this term is uniform due to the scale separation. A change in variable may be useful: if we denote

$$
\theta_{F 0}(x, y, t)=\theta_{S 0}(x, t)+w(x, y, t)
$$

the new problem consists in finding $w$ which is $\Omega$-periodic, null on $\Gamma$, such that

$$
\rho_{F} c_{F} \dot{\theta}_{F 0}+\rho_{F} c_{F} \dot{w}=\operatorname{div}_{y}\left(k_{F}^{\prime} \underline{\operatorname{grad}}_{y} w\right) \text { in } \Omega_{F}
$$

Using a test function which is $\Omega$-periodic and null on $\Gamma$, the variational formulation reads:

$$
\int_{\Omega_{F}} w^{\star} \rho_{F} c_{F} \dot{w} d \Omega+\int_{\Omega_{F}} \underline{\operatorname{grad}} w^{\star} \cdot k_{F}^{\prime} \underline{\operatorname{grad}}_{y} w d \Omega==-\int_{\Omega_{F}} w^{\star} \rho_{F} c_{F} \dot{\theta}_{F 0} d \Omega
$$




\subsection{A macro model with a 2-field temperature, or with a memory function}

The macroscopic model is obtained by ensuring that the next higher order problem for $\theta_{S 2}$ is well-posed. It reads:

$$
\begin{array}{r}
-\operatorname{div}_{y} \underline{q}_{S 1}-\operatorname{div}_{x} \underline{q}_{S 0}=\rho_{S} c_{S} \dot{\theta}_{S 0} \quad \text { in } \Omega_{S} \\
\underline{q}_{S 1} \cdot \underline{n}_{S}+\underline{q}_{F 1} \cdot \underline{n}_{F}=0 \text { on } \Gamma \\
\underline{q}_{S 1}=-k_{S}\left(\underline{\operatorname{grad}}_{x} \theta_{S 1}+\underline{\operatorname{grad}}_{y} \theta_{S 2}\right) \text { in } \Omega_{S} \\
\theta_{S 2} \text { is } \Omega \text {-periodic }
\end{array}
$$

The partial averaging operations are defined as $\langle\bullet\rangle_{S}=\frac{1}{\operatorname{vol}(\Omega)} \int_{\Omega_{S}} \bullet d \Omega$ and $\langle\bullet\rangle_{F}=\frac{1}{\operatorname{vol}(\Omega)} \int_{\Omega_{F}} \bullet d \Omega$, so that $\frac{1}{\operatorname{vol}(\Omega)} \int_{\Omega} \bullet d \Omega=\langle\bullet\rangle_{S}+\langle\bullet\rangle_{F}$. The compatibility condition is obtained by averaging the first equation (and using periodicity), leading to [7]:

$$
-\frac{1}{\operatorname{vol}(\Omega)} \int_{\Gamma} \underline{q}_{S 1} \cdot \underline{n}_{S} d \Gamma+\operatorname{div}_{x}\left(\left\langle k_{S}\left(\mathbf{1}-\boldsymbol{L}_{S}\right)\right\rangle_{S} \underline{Z}_{S 0}\right)=\left\langle\rho_{S} c_{S}\right\rangle_{S} \dot{\theta}_{S 0}
$$

with the macroscopic parameters

$$
C_{M S}=\left\langle\rho_{S} c_{S}\right\rangle_{S} \quad \text { and } \quad \boldsymbol{k}_{M S}=\left\langle k_{S}\left(\mathbf{1}-\boldsymbol{L}_{S}\right)\right\rangle_{S}
$$

The average of the previous problem giving $\theta_{F 0}$ reads:

$$
\begin{aligned}
-\frac{1}{\operatorname{vol}(\Omega)} \int_{\Gamma} \underline{q}_{F 1} \cdot \underline{n}_{F} d \Gamma & =\left\langle\rho_{F} c_{F} \dot{\theta}_{F 0}\right\rangle_{F} \\
& =\frac{1}{\operatorname{vol}(\Omega)} \int_{\Gamma} \underline{q}_{S 1} \cdot \underline{n}_{S} d \Gamma
\end{aligned}
$$

Finally, the macroscopic problem reads:

$$
C_{M S} \dot{\theta}_{S 0}=\operatorname{div}_{x}\left(\boldsymbol{k}_{M S} \underline{Z}_{S 0}\right)-\left\langle\rho_{F} c_{F} \dot{\theta}_{F 0}\right\rangle_{F}
$$

It also involves the second thermal field rate $\dot{\theta}_{F 0}$ defined on the microstructure.

This problem can be seen as a macroscopic thermal problem with additional internal variables at each macro material point which are the underlying values of $\dot{\theta}_{F 0}$ on a full micro cell. Once discretized by finite elements, this involves at each macro integration point the storage of a full field $\dot{\theta}_{F 0}$ at each nodes of the underlying discretized cell, as used in the so-called $\mathrm{FE}^{2}$ approach [14]. In our context, this approach is too storage consuming. Note that in the past, memory effect models were designed for visco-elasticity problems, but have been superseded by models with internal variables [1]. The converse way is used herein, since we promote an available memory effect model [7]:

$$
C_{M S} \dot{\theta}_{S 0}=\operatorname{div}_{x}\left(\boldsymbol{k}_{M S} \underline{Z}_{S 0}\right)+\int_{\tau=-\infty}^{\tau=t} \ddot{\theta}_{S 0}(\tau) \beta(t-\tau) d \tau
$$

where $\beta(t)$ is a memory function, that should depend only on the macro material (independent of boundary conditions) and that should vanish for large $t$ (evanescent memory). Concerning practical initial value for this problem, we may consider that a stationary state is given for $t \leq 0$, i.e. $\dot{\theta}_{S 0}(t \leq 0)=0$ and $\dot{\theta}_{F 0}(t \leq 0)=0$, so that the back-in-time can start for $\tau=0$, and we may define the searched temperature differences as the differences with respect to these initial temperatures, so that one also has: $\theta_{S 0}(t \leq 0)=0$ and $\theta_{F 0}(t \leq 0)=0$.

The results given in [7] and reused previously are established using a Fourier transform method. This approach also enabled the author to derive the memory function as an infinite sum of a series, for a simple 1D problem. In this article, we aim to establish the possibility of computing the memory function in a general way, using only transient problems on a unique cell, in a similar fashion as the two other homogenized parameters $C_{M S}$ and $\boldsymbol{k}_{M S}$ that are derived from stationary simulations on the same unique cell.

\section{Numerical determination of macro material parameters}

As mentioned before, the parameters $C_{M S}$ and $\boldsymbol{k}_{M S}$ can be computed in a standard fashion, solving simple steady state problems on a single cell with periodic boundary conditions. The last parameter is now the memory function $\beta$ to be determined. 


\subsection{Discretization of the memory effect}

The first step is the discretized-in-time version of the memory effect. We propose to use a simple implicit Euler integration scheme. Note that the same procedure can be used for any other scheme. We denote $t_{k}$ the time instant number $k$, and $h$ the time step (assumed constant for sake of simplicity), and we use the superscript or the subscript $k$ to denote the value of a quantity at time $t_{k}$. Moreover the considered instant for unknown quantities is $t_{i+1}$, and the past instants are $t_{k}$ for $k=0, \ldots, i$; by convention, $t_{0}=0$.

The time integration scheme relates the values for the derivative of a quantity $w$ to the values of the same quantity. Here it reads: $w_{i+1}=w_{i}+h \dot{w}_{i+1}$ or equivalently $\dot{w}_{i+1}=\frac{1}{h}\left(w_{i+1}-w_{i}\right)$.

Concerning the heredity integral with the second derivative of the micro temperature field, dealing with first order derivatives is easier for a time integration scheme dedicated to parabolic equations. We therefore discretize the integral using only values for the first order derivative of the temperature. To do so, we integrate by parts to formally use the derivative of the memory function $\gamma(t)=\dot{\beta}(t)$, and the time integration scheme:

$$
\begin{aligned}
\int_{0}^{t} \ddot{\theta}_{S 0}(\tau) \beta(t-\tau) d \tau & =\dot{\theta}_{S 0}(t) \beta(0)-\dot{\theta}_{S 0}(0) \beta(t)+\sum_{k=0}^{i} \int_{t_{k}}^{t_{k+1}} \dot{\theta}_{S 0}(\tau) \gamma\left(t_{i+1}-\tau\right) d \tau \\
& \approx \dot{\theta}_{S 0}^{i+1} \beta_{0}-\dot{\theta}_{S 0}^{0} \beta_{i+1}+\sum_{k=0}^{i} \dot{\theta}_{S 0}^{i+1-k} \gamma_{k} h
\end{aligned}
$$

and $\beta_{k}=\beta_{k-1}+h \gamma_{k}$.

\subsection{Discretization of the micro problem of the $F$-phase}

On a second hand, we wish to express the solution of the transient thermal problem on the $F$-cell with a recursion formula (in a back-in-time expression). To do so, we consider a spatial finite element discretization of the corresponding variational problem. It reads at time $t_{i+1}$ :

$$
\boldsymbol{w}^{\star T}\left(\boldsymbol{C} \dot{\boldsymbol{w}}_{i+1}+\boldsymbol{K} \boldsymbol{w}_{i+1}\right)=-\boldsymbol{w}^{\star T} \boldsymbol{C u} \dot{\theta}_{S 0}^{i+1}
$$

$\boldsymbol{C}$ and $\boldsymbol{K}$ are the capacity and conductivity matrices using the material parameters $\rho_{F} c_{F}$ and $k_{F}^{\prime}$ on the $\Omega_{F}$ domain, $\boldsymbol{w}$ is the column vector of nodal values for the thermal field $w, \boldsymbol{u}$ is a unitary vector column of nodal values, so that $\boldsymbol{u} \dot{\theta}_{S 0}^{i+1}$ is a discretized uniform field with value $\dot{\theta}_{S 0}^{i+1}$. The thermal field $w$ should be $\Omega$-periodic and null on $\Gamma$, therefore the corresponding column vector can be built from a smaller size vector, $\widetilde{\boldsymbol{w}}$, getting rid of null values on the nodes on $\Gamma$ and dependent values due to periodicity. Formally, we may use a boolean rectangular mapping matrix $\boldsymbol{B}$ (with $\boldsymbol{B} \boldsymbol{B}^{T}$ being the identity on a reduced subspace) to express these dependencies as $\boldsymbol{w}=\boldsymbol{B}^{T} \widetilde{\boldsymbol{w}}$; so does the test functions: $\boldsymbol{w}^{\star}=\boldsymbol{B}^{T} \widetilde{\boldsymbol{w}}^{\star}$. Therefore, the problem reads

$$
\left(\boldsymbol{B} \boldsymbol{C} \boldsymbol{B}^{T}\right) \dot{\widetilde{\boldsymbol{w}}}_{i+1}+\left(\boldsymbol{B} \boldsymbol{K} \boldsymbol{B}^{T}\right) \widetilde{\boldsymbol{w}}_{i+1}=-\boldsymbol{B} \boldsymbol{C u} \dot{\theta}_{S 0}^{i+1}
$$

Using now the time integration scheme, it transforms into:

$$
\left(\boldsymbol{B} \frac{1}{h} \widetilde{\boldsymbol{C}} \boldsymbol{B}^{T}\right) \widetilde{\boldsymbol{w}}_{i+1}=-\boldsymbol{B} \boldsymbol{C u} \dot{\theta}_{S 0}^{i+1}+\frac{1}{h} \boldsymbol{B} \boldsymbol{C} \boldsymbol{w}_{i}
$$

where $\widetilde{\boldsymbol{C}}=\boldsymbol{C}+h \boldsymbol{K}$ is invertible, so one gets the expression of $\widetilde{\boldsymbol{w}}_{i+1}$, then of $\boldsymbol{w}_{i+1}=\boldsymbol{B}^{T} \widetilde{\boldsymbol{w}}_{i+1}$ :

$$
\boldsymbol{w}_{i+1}=\boldsymbol{A} \boldsymbol{w}_{i}-h \boldsymbol{A} \boldsymbol{u} \dot{\theta}_{S 0}^{i+1}
$$

with

$$
\boldsymbol{A}=\boldsymbol{B}^{T}\left(\boldsymbol{B} \widetilde{\boldsymbol{C}} \boldsymbol{B}^{T}\right)^{-1} \boldsymbol{B} \boldsymbol{C}
$$

Using this recursion formula for $\boldsymbol{w}$ together with the time integration scheme allows to find the recursion formula for $\dot{\boldsymbol{w}}$ as well, leading after algebraic manipulations to:

$$
\dot{\boldsymbol{w}}_{i+1}=\boldsymbol{A}^{i}(\boldsymbol{A}-\mathbf{1}) \frac{1}{h} \boldsymbol{w}_{0}-\sum_{k=1}^{i} \boldsymbol{A}^{k}(\boldsymbol{A}-\mathbf{1}) \boldsymbol{u} \dot{\theta}_{S 0}^{i+1-k}-\boldsymbol{A u} \dot{\theta}_{S 0}^{i+1}
$$


Noting that $w_{0}=\theta_{F 0}^{0}-\theta_{S 0}^{0}=0$, one finally gets

$$
\dot{\boldsymbol{\theta}}_{F 0}^{i+1}=-\sum_{k=0}^{i} \boldsymbol{A}^{k}(\boldsymbol{A}-\mathbf{1}) \boldsymbol{u} \dot{\theta}_{S 0}^{i+1-k}
$$

Note that for a parabolic equation of a time-dependent problem such as this micro problem, spurious but bounded oscillations can appear for a too short time step due to the non satisfaction of the discrete maximum principle $[15,16]$. The rule-of-thumb (obtained for a discretized $1 \mathrm{D}$ problem) is that $[17,18]$ $h \geq\left(\rho_{F} c_{F} e^{2}\right) /\left(6 k_{F}^{\prime} \alpha\right)$ where $e$ is the element size and $\alpha$ the parameter for the $\theta$-method time integration scheme: here, $\alpha=1$ for implicit Euler method. Therefore, the bound can be stated as

$$
\frac{h}{\tau_{F}} \geq \frac{\pi^{2}}{6}\left(\frac{e}{L_{F}}\right)^{2}=\frac{\pi^{2}}{6}\left(\frac{e}{l_{F}}\right)^{2} \varepsilon^{2}
$$

Otherwise, a lumping of the capacity matrix (or a lower order estimates) allows to remove the bound [19], for instance using a numerical integration with integration points located at nodes for the capacity model. In the following, we will always satisfy the previous criteria.

\subsection{Identification of the two models}

Identifying the two previous discretized versions, (7) and (10), of the same term in (5) and (6):

$$
\int_{\tau=0}^{\tau=t} \ddot{\theta}_{S 0}(\tau) \beta(t-\tau) d \tau=-\left\langle\rho_{F} c_{F} \dot{\theta}_{F 0}\right\rangle_{F}
$$

leads to:

$$
\begin{array}{r}
\beta_{0}-h \gamma_{0}=-\left\langle\rho_{F} c_{F}(\mathbf{1}-\boldsymbol{A}) \boldsymbol{u}\right\rangle_{F} \\
h \gamma_{k}=\left\langle\rho_{F} c_{F} \boldsymbol{A}^{k}(\boldsymbol{A}-\mathbf{1}) \boldsymbol{u}\right\rangle_{F} \quad \text { for } k=1, \ldots, i
\end{array}
$$

There is still an indeterminacy for $\beta_{0}$ and $\gamma_{0}$ that can be overcome by ensuring the vanishing property of the memory function $\beta$. Indeed, using the incremental relationship $\beta_{k+1}=\beta_{k}+h \gamma_{k+1}$, one gets

$$
\beta_{i+1}=\beta_{0}+\sum_{k=1}^{i+1} h \gamma_{k}=\beta_{0}-\left\langle\rho_{F} c_{F}\left(\mathbf{1}-\boldsymbol{A}^{i+1}\right) \boldsymbol{A} \boldsymbol{u}\right\rangle_{F}
$$

If the spectral radius of the iterated operator $\boldsymbol{A}$ is $\rho(\boldsymbol{A})<1$, then the limit of $\beta_{i+1}$ for large values of $i$ is $\beta_{0}-\left\langle\rho_{F} c_{F} \boldsymbol{A} \boldsymbol{u}\right\rangle_{F}$. Nullifying this value for a vanishing memory function leads to choose

$$
\beta_{0}=\left\langle\rho_{F} c_{F} \boldsymbol{A} \boldsymbol{u}\right\rangle_{F}=\frac{1}{\operatorname{vol}(\Omega)} \boldsymbol{u}^{T} \boldsymbol{C A} \boldsymbol{u}
$$

so that for $k=0, \ldots, i$

$$
\beta_{k}=\left\langle\rho_{F} c_{F} \boldsymbol{A}^{k+1} \boldsymbol{u}\right\rangle_{F}=\frac{1}{\operatorname{vol}(\Omega)} \boldsymbol{u}^{T} \boldsymbol{C} \boldsymbol{A}^{k+1} \boldsymbol{u}
$$

A rough proof of the contraction property of the operator $\boldsymbol{A}$ is the following: within the subspace of $\Omega$-periodic thermal fields that are null on $\Gamma$, consider any thermal field $\boldsymbol{w} \neq 0$ and denote $\boldsymbol{v}=\boldsymbol{A} \boldsymbol{w}$ with $\boldsymbol{A}=\widetilde{\boldsymbol{C}}^{-1} \boldsymbol{C}$ and $\widetilde{\boldsymbol{C}}=\boldsymbol{C}+h \boldsymbol{K}(\boldsymbol{C}$ is symmetric definite positive, and $\boldsymbol{K}$ is symmetric positive, and definite on the considered subspace, provided that the measure of $\Gamma$ is not null, i.e. that the phases are not separated). One has

$$
\begin{aligned}
(\boldsymbol{w}-\boldsymbol{v})^{T} \boldsymbol{C}(\boldsymbol{w}-\boldsymbol{v}) & =\boldsymbol{w}^{T} \boldsymbol{C} \boldsymbol{w}+\boldsymbol{v}^{T} \boldsymbol{C} \boldsymbol{v}-2 \boldsymbol{v}^{T} \boldsymbol{C} \boldsymbol{w} \\
& =\boldsymbol{w}^{T} \boldsymbol{C} \boldsymbol{w}+\boldsymbol{v}^{T} \boldsymbol{C} \boldsymbol{v}-2 \boldsymbol{v}^{T}(\boldsymbol{C}+h \boldsymbol{K}) \boldsymbol{v} \\
& =\boldsymbol{w}^{T} \boldsymbol{C} \boldsymbol{w}-\boldsymbol{v}^{T} \boldsymbol{C} \boldsymbol{v}-2 h \boldsymbol{v}^{T} \boldsymbol{K} \boldsymbol{v}
\end{aligned}
$$

We can therefore conclude that $\boldsymbol{v}^{T} \boldsymbol{C} \boldsymbol{v}=\boldsymbol{w}^{T} \boldsymbol{C} \boldsymbol{w}-(\boldsymbol{w}-\boldsymbol{v})^{T} \boldsymbol{C}(\boldsymbol{w}-\boldsymbol{v})-2 h \boldsymbol{v}^{T} \boldsymbol{K} \boldsymbol{v}$. Provided that $\boldsymbol{v} \neq \boldsymbol{w}$, one gets $\boldsymbol{v}^{T} \boldsymbol{C} \boldsymbol{v}<\boldsymbol{w}^{T} \boldsymbol{C} \boldsymbol{w}$. Assume now that $\boldsymbol{w}$ is an eigenvector of $\boldsymbol{A}$ associated to the eigenvalue $\lambda \neq 1: \boldsymbol{v}=\lambda \boldsymbol{w}$ and the previous inequality leads to $\lambda^{2} \boldsymbol{w}^{T} \boldsymbol{C} \boldsymbol{w}<\boldsymbol{w}^{T} \boldsymbol{C} \boldsymbol{w}$, so $\lambda^{2}<1$. To complete the 
proof, consider the case where $\lambda=1$ and $\boldsymbol{v}=\boldsymbol{w}$; the previous developments allow to conclude that $\boldsymbol{v}^{T} \boldsymbol{K} \boldsymbol{v}=0$ and therefore (on the dedicated subspace) $\boldsymbol{v}=0$, so $\boldsymbol{w}=0$ which is contradictory, so $\lambda \neq 1$. The final conclusion is therefore: $\rho(\boldsymbol{A})<1$.

The simple overall pseudo-code to build the values of the memory function is summarized in the algorithm 1. Since this memory function is interpreted as a macroscopic homogenized material characteristics, it is determined with computations at the cell microstructure level, as the other homogenized material parameters are, such as macroscopic conductivity and macroscopic capacity.

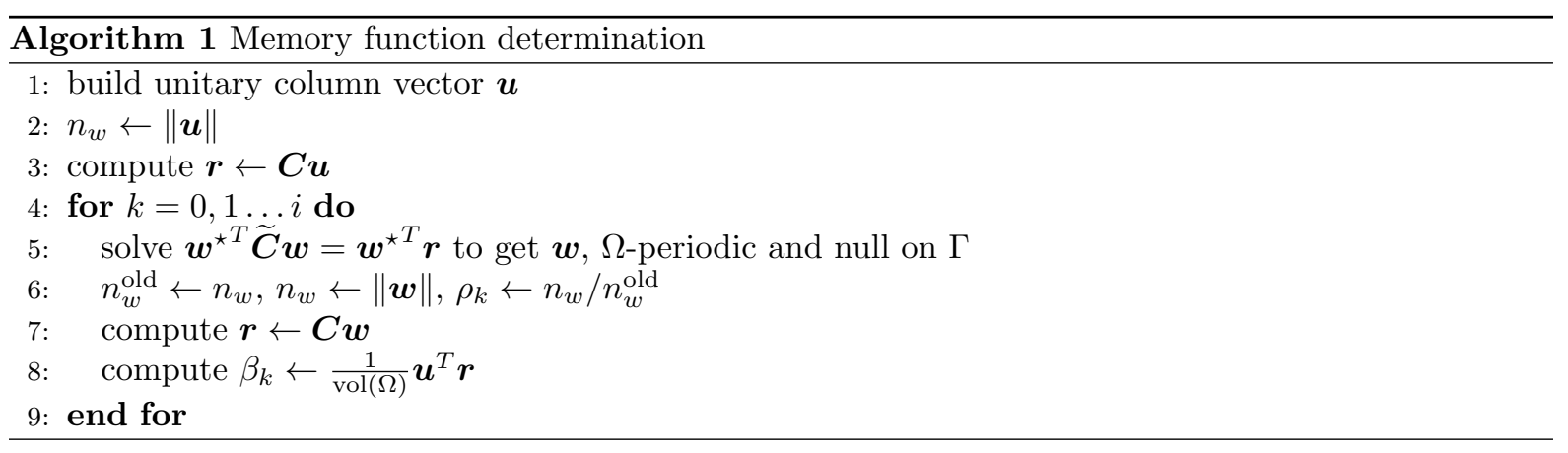

Note that the characteristic time $\tau_{F 0}$ for the discretized-in-space problem is linked to the previous operators in the left hand side. Indeed, one can defined it as being the inverse of the minimal eigenvalue of $\left(\boldsymbol{B} \boldsymbol{C} \boldsymbol{B}^{T}\right)^{-1}\left(\boldsymbol{B} \boldsymbol{K} \boldsymbol{B}^{T}\right)$. So, the spectral radius of $\boldsymbol{A}$ is $\rho(\boldsymbol{A})=\left(1+h / \tau_{F 0}\right)^{-1}<1$ and

$$
\tau_{F 0}=\frac{h}{\rho(\boldsymbol{A})^{-1}-1}
$$

More precisely, the restriction of $\boldsymbol{A}$ in the reduced subspace of admissible fields is

$$
\boldsymbol{B} \boldsymbol{A} \boldsymbol{B}^{T}=\mathbf{1}+h\left(\boldsymbol{B} \boldsymbol{C} \boldsymbol{B}^{T}\right)^{-1}\left(\boldsymbol{B} \boldsymbol{K} \boldsymbol{B}^{T}\right)
$$

Usually, the matrix $\boldsymbol{A}$ is not explicitly available, but its spectral radius can be obtained with the iterates of the power method. This is also what is done in the algorithm 1 for two successive vectors $\boldsymbol{w}_{k}$ and $\boldsymbol{w}_{k+1}$ : when sufficiently iterated, the spectral radius is close to $\rho_{k+1}=\left\|\boldsymbol{w}_{k+1}\right\| /\left\|\boldsymbol{w}_{k}\right\|$. The stabilization of this value therefore denotes that the evolution of the function $\beta$ is found for "large" values of $t / \tau_{F}$ therefore leading to an exponential decay for $\beta(t)$ of the form $\exp \left(-t / \tau_{F 0}\right)$.

\subsection{Memory effects on the different macroscopic fields}

The previous developments consider $\theta_{S 0}$ as a macroscopic temperature field. Indeed, it is independent of the fast coordinate. For the other phase, an average of the temperature is also a macroscopic field, i.e. $\left\langle\theta_{F 0}\right\rangle_{F}$, as well as the average of the first temperature terms of both phases, i.e.

$$
\bar{\theta}=\left\langle\theta_{S 0}\right\rangle_{S}+\left\langle\theta_{F 0}\right\rangle_{F}=(1-n) \theta_{S 0}+\left\langle\theta_{F 0}\right\rangle_{F}
$$

where $n$ is the fraction of $F$-phase.

Considering the recurrence formula (10) (and its counterpart with no time derivative), one gets the linear combinations

$$
\begin{aligned}
& \left\langle\dot{\theta}_{F 0}^{i+1}\right\rangle_{F}=\sum_{k=0}^{i} \alpha_{k} \dot{\theta}_{S 0}^{i+1-k} \quad \text { and } \\
& \left\langle\theta_{F 0}^{i+1}\right\rangle_{F}=\sum_{k=0}^{i} \alpha_{k} \theta_{S 0}^{i+1-k}
\end{aligned}
$$

with $\alpha_{k}=-\left\langle\boldsymbol{A}^{k}(\boldsymbol{A}-\mathbf{1}) \boldsymbol{u}\right\rangle_{F}$.

In the special case where the microstructure is identically the same at any macroscopic point, and when $\rho_{F} c_{F}$ is constant, the macroscopic problem (5), can be written at the different time steps $i+1-k$ and weighted with the coefficients $\alpha_{k}$ to give

$$
\alpha_{k} C_{M S} \dot{\theta}_{S 0}^{i+1-k}=\operatorname{div}_{x}\left(\boldsymbol{k}_{M S} \underline{\operatorname{grad}}_{x}\left(\alpha_{k} \theta_{S 0}^{i+1-k}\right)\right)-\rho_{F} c_{F} \alpha_{k}\left\langle\dot{\theta}_{F 0}^{i+1-k}\right\rangle_{F}
$$




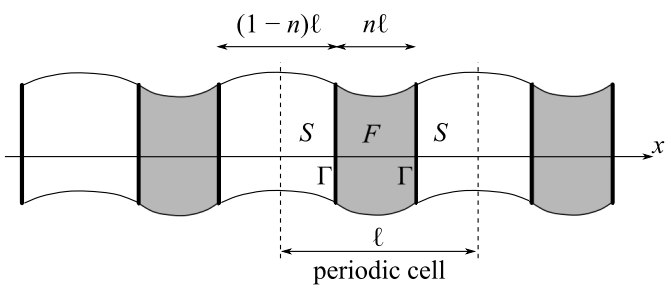

Figure 1: 1D test case of a bilaminated composite (infinite in vertical direction, periodic in horizontal direction).

leading when summing for $k=0, \ldots, i$ to:

$$
C_{M S}\left\langle\dot{\theta}_{F 0}^{i+1}\right\rangle_{F}=\operatorname{div}_{x}\left(\boldsymbol{k}_{M S} \underline{\operatorname{grad}}_{x}\left\langle\theta_{F 0}^{i+1}\right\rangle_{F}\right)-\sum_{k=0}^{i} \rho_{F} c_{F} \alpha_{k}\left\langle\dot{\theta}_{F 0}^{i+1-k}\right\rangle_{F}
$$

whereas the first macroscopic problem (5) was

$$
C_{M S} \dot{\theta}_{S 0}^{i+1}=\operatorname{div}_{x}\left(\boldsymbol{k}_{M S} \underline{\operatorname{grad}}_{x} \theta_{S 0}^{i+1}\right)-\sum_{k=0}^{i} \rho_{F} c_{F} \alpha_{k} \dot{\theta}_{S 0}^{i+1-k}
$$

i.e. with the same heredity term function in the right-hand-side. As a consequence, the macroscopic problem for the total average temperature is also of the same form:

$$
C_{M S} \dot{\bar{\theta}}^{i+1}=\operatorname{div}_{x}\left(\boldsymbol{k}_{M S} \underline{\operatorname{grad}}_{x} \bar{\theta}^{i+1}\right)-\sum_{k=0}^{i} \rho_{F} c_{F} \alpha_{k} \dot{\bar{\theta}}^{i+1-k}
$$

\section{Numerical examples}

\subsection{Bilaminated composite}

This 1D test case is defined in [7] for which a Fourier analysis allows to have an explicit expression for the memory function ${ }^{1}$. It concerns a stack of 2 kinds of layers $(S$ and $F)$ with a volumic fraction $n$ of phase $F$, see figure 1 . This example may serve as a validation test for the proposed approach to compute numerically the memory function, as well as to illustrate the dependence on the discretization parameters, i.e. the time step $h$ and the finite element size $e$.

When the macroscopic temperature is considered constant, but a function of a parameter $\tau$, a Fourier analysis gives the particular expression of the searched temperature for phase $F$ as $\Phi(x, t ; \tau)=$ $\phi(x, t) \theta_{S 0}(\tau)$ with

$$
\begin{aligned}
\phi(x, t) & =-\sum_{q=0}^{\infty} u_{q}(x) v_{q}(t) \\
u_{q}(x) & =\frac{4}{(2 q+1) \pi} \sin \left[(2 q+1) \pi x / L_{F}\right] \\
v_{q}(t) & =\exp \left[-(2 q+1)^{2} t / \tau_{F}\right]
\end{aligned}
$$

To find the solution when the macroscopic temperature depends on time $t$, the use of Duhamel integral provides

$$
w(x, t)=\frac{\partial}{\partial t} \int_{0}^{t} \Phi(x, t-\tau ; \tau) d \tau
$$

With an initial condition $\theta_{S 0}(x, 0)=0$, and $v_{q}(0)=0$, this leads to

$$
\begin{aligned}
w(x, t) & =\int_{0}^{t} \dot{\theta}_{S 0}(\tau) \phi(x, t-\tau) d \tau \\
\dot{w}(x, t) & =\dot{\theta}_{S 0}(0) \phi(x, t)+\int_{0}^{t} \ddot{\theta}_{S 0}(\tau) \phi(x, t-\tau) d \tau \\
\dot{\theta}_{F 0}(x, t) & =\dot{w}(x, t)+\dot{\theta}_{S 0}(t)
\end{aligned}
$$

\footnotetext{
${ }^{1}$ a typo in the original paper is corrected here, so intermediate steps are provided herein to obtain the analytical solution.
} 
so that

$$
\begin{aligned}
-\left\langle\rho_{F} c_{F} \dot{\theta}_{F 0}(x, t)\right\rangle_{F}=\int_{0}^{t} \ddot{\theta}_{S 0}(\tau) \sum_{q=0}^{\infty}\left\langle\rho_{F} c_{F} u_{q}(x)\right\rangle_{F} & v_{q}(t-\tau) d \tau+ \\
& +\dot{\theta}_{S 0}(0) \sum_{q=0}^{\infty}\left\langle\rho_{F} c_{F} u_{q}(x)\right\rangle_{F} v_{q}(t)-\rho_{F} c_{F} n \dot{\theta}_{S 0}(t)
\end{aligned}
$$

Hence

$$
\begin{aligned}
\beta(t) & =\sum_{q=0}^{\infty}\left\langle\rho_{F} c_{F} u_{q}(x)\right\rangle_{F} v_{q}(t) \\
& =\rho_{F} c_{F} n \frac{8}{\pi^{2}} \sum_{q=0}^{\infty} \frac{1}{(2 q+1)^{2}} \exp \left[-(2 q+1)^{2} t / \tau_{F}\right]
\end{aligned}
$$

for which

$$
\beta(0)=\rho_{F} c_{F} n \frac{8}{\pi^{2}} \sum_{q=0}^{\infty} \frac{1}{(2 q+1)^{2}}=\rho_{F} c_{F} n
$$

If only one term of the development is kept for an approximate analytical solution, it reads:

$$
\beta(t) \approx \rho_{F} c_{F} n \frac{8}{\pi^{2}} \exp \left[-t / \tau_{F}\right]
$$

These solutions are depicted on Figure 2.

For validation purposes, a $1 \mathrm{D}$ discretization of $\Omega_{F}=\left[0, L_{F}=n L\right]$ is done with $n_{e}$ 2-node finite elements of uniform size $e=n L / n_{e}$, and a time discretization of $\left[0, \tau_{F}\right]$ with $n_{h}$ time steps, also with a uniform time step $h=\tau_{F} / n_{h}$. The error with respect to the exact solution is depicted on figure 3 . It is concentrated at the beginning of the time evolution of $\beta(t)$. This is due to the fact that the elementary solution that is to be captured by the numerical method is a somehow thermal boundary layer (boundary conditions with a different value than the initial condition), for which a fine discretization is useful to capture the good average. Such boundary layers can be captured using mesh local refinements or with enriched FE methods [20,21]. Here, we focus on a semi-analytical approximation to get the first values of the memory effect function $\beta(t)$ for the beginning of its evolution, i.e. for a "small" time $t$. Indeed, analytical solutions are available for an infinite half plane, i.e. locally to each point of the boundary $\Gamma$ when the boundary layer is close to the boundary: in the direction of the normal to the surface, with a coordinate $x$, it is $\phi(x, t) \approx \operatorname{erf}(u)$; erf is the error function, and

$$
u=\frac{x}{\sqrt{\left(4 k_{F}^{\prime} / \rho_{F} c_{F}\right) t}}=\frac{\pi}{2} \frac{x / L_{F}}{\sqrt{t / \tau_{F}}}
$$

Using a similar development as before, this allows to get an approximate solution for the memory function:

$$
\begin{aligned}
\beta(t) & \approx \rho_{F} c_{F} \frac{1}{\operatorname{vol}(\Omega)} \int_{\Gamma} \int_{0}^{\widetilde{L}} \phi(x, t) d x d S \\
& =\rho_{F} c_{F} \frac{\operatorname{surf}(\Gamma)}{\operatorname{vol}(\Omega)} \int_{0}^{\widetilde{L}} \phi(x, t) d x
\end{aligned}
$$

$\widetilde{L}$ is a normal distance to the boundary, allowing to have a significant integral on the whole domain $\Omega_{F}$. For the previous $1 \mathrm{D}$ problem, there is no surface integral but two boundaries, and $\widetilde{L}=L_{F} / 2$ (each boundary is associated to half of the domain). For 3D problems, and for consistency reasons (exact integration of a constant field), one can choose

$$
\widetilde{L}=\operatorname{vol}\left(\Omega_{F}\right) / \operatorname{surf}(\Gamma)=n \times \operatorname{vol}(\Omega) / \operatorname{surf}(\Gamma)
$$

An analytic expression of the integral is available, so that

$$
\beta(t) \approx \rho_{F} c_{F} \frac{\operatorname{surf}(\Gamma) \widetilde{L}}{\operatorname{vol}(\Omega)}\left[\operatorname{erf}\left(\frac{1}{\epsilon}\right)+\frac{\epsilon}{\sqrt{\pi}}\left(\exp \left(-\frac{1}{\epsilon^{2}}\right)-1\right)\right]
$$




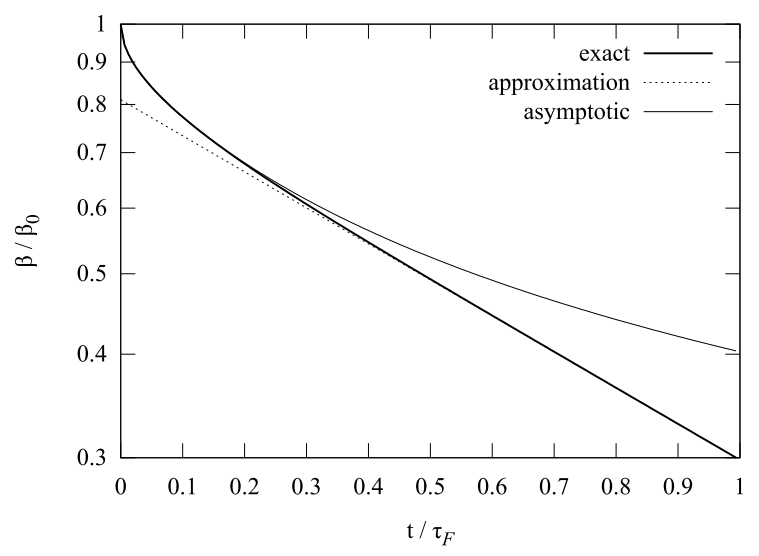

Figure 2: Analytical results for the 1D test case of a bilaminated composite.

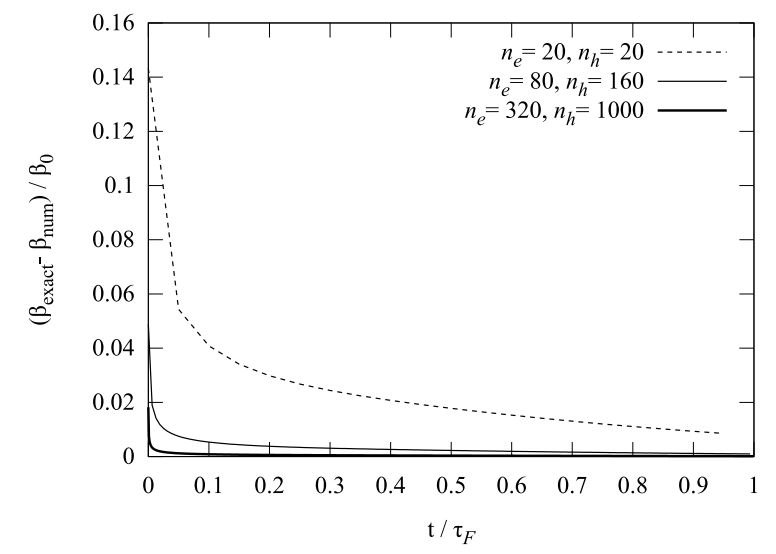

Figure 3: Error between the exact solution and the numerically obtained solution with different uniform discretizations.

with

$$
\epsilon=\frac{1}{\widetilde{L}} \sqrt{\frac{4 k_{F}^{\prime}}{\rho_{F} c_{F}}} t=\frac{2}{\pi} \frac{\sqrt{t / \tau_{F}}}{\widetilde{L} / L_{F}}
$$

which is expected to be accurate for $\epsilon$ being small. This asymptotic solution is also depicted on figure 2 for the 1D test case, for which $\operatorname{surf}(\Gamma) \widetilde{L} / \operatorname{vol}(\Omega)$ is replaced with $n=L_{F} / L$. Indeed, we checked that the approximation (16) fits the exact solution at small time values (say for $t / \tau_{F} \leq 0.2$ ), while the exponential decay approximation is correct for large time values (say for $t / \tau_{F} \geq 0.4$ ).

\subsection{A 3D connected phase with low conductivity}

For 3D problems, approximate close forms of the solution - asymptotic solution (16) and the exponential decay $\exp \left(-t / \tau_{F 0}\right)$ - are therefore driven by the correct choices of characteristic length $\widetilde{L}$ and characteristic time $\tau_{F 0}$. Nevertheless, for a complex microstructure, their evaluation may not be trivial, so the previous transient numerical simulation on the microstructure is still of interest.

The proposed test case is depicted on figure 4 . The $F$-phase is arranged along a cubic connected lattice, and the $S$-phase is the matrix which surrounds. In the proposed case, with a scalar temperature field as unknown, only $1 / 8$ of a cell can be simulated, discarding the periodicity conditions that are automatically satisfied due to the planar symmetries. The fraction of $F$-phase is $n=0.354$.

Since the spectral radius of $\boldsymbol{A}$ is $\left(1+h / \tau_{F 0}\right)^{-1}$, the smaller $h$ is, the slower the iterative power method converges (i.e. the simulation gives the initial evolution of $\beta(t)$, not the asymptotic convergence rate when $t$ is large). Therefore, an initial computation can be done with a somehow large time step $h$, not to provide the accurate memory function evolution, but to reach the exponential decay regime, to give the value of $\tau_{F 0}$ (depending on the spatial mesh size, but not on the time step). Then, a second simulation with a small time step will give the evolution of $\beta(t)$ for small time $t$. Eventually, this last computation 


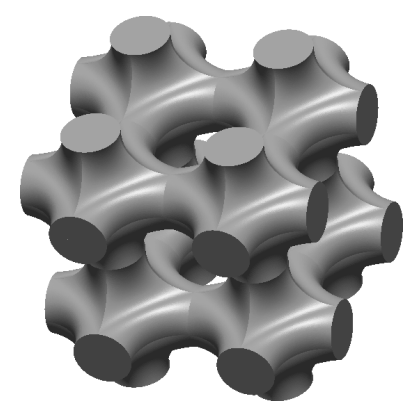

Figure 4: Material microstructure for phase $F$. A group of 8 cells is depicted.
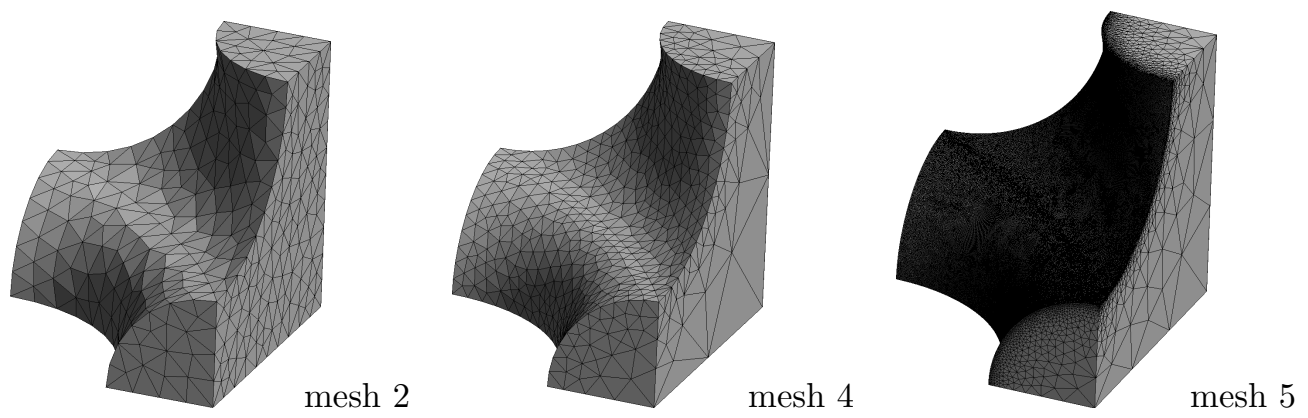

Figure 5: Different spatial discretizations of $1 / 8$ th of the cell.

can be replaced with the approximation of the memory function for small time $t$ (16); this requires a guess for the characteristic length $\widetilde{L}$, that can be given with (15).

In the following tests, we first compute a characteristic time value $\tau_{F 0}$ with a coarse time step and different mesh sizes to assess the precision of the estimate. Second, we run several simulations with different fine time steps and mesh sizes to study the convergence to the memory function, and compare the results with the previous approximation (16) for small time values. All pre and post-processings have been done with Gmsh [22] and Paraview [23] codes, and processing with the FE code Cast3M [24].

A first set of simulations is performed with 40 time steps only on a time interval $\left[0,10 \tau_{F}\right]$, with an estimated $\tau_{F}$ obtained with a length $L_{F}$ taken as 0.4 times the cell edge length. Several meshes (with P1 4-node tetrahedral elements) are used: regular meshes with different refinement levels (meshes 1 to 3 ), and a mesh with a size gradient (mesh 4), the smallest elements being located on the interface $\Gamma$, figure 5. The obtained values as well as the meshes characteristics are reported in table 1, and time evolutions are plot on figure 6 . The case of the overkilling mesh 3 is considered as the reference. Clearly, the mesh with a size gradient is not the most suited to estimate the exponential decay; this is due to the fact that at a long term evolution, the temperature field (i.e. the mode associated to the eigenvalue giving the spectral radius of $\boldsymbol{A}$ ) is no more a boundary layer, but spreads on the whole cell, figure 7 . A uniform mesh is therefore suited to capture it.

The second set of simulation is performed along a shorter time interval but with a much fine time discretization, also reported in table 1 . The obtained memory functions are depicted on figure 8 .

The result obtained with mesh 5 is considered as a reference. For this simulation, an adapted mesh such as mesh 4 is now suited to capture the solution, figure 7 . The asymptotic solution for small time values is still a good approximation of the reference solution up to $t / \tau_{F} \leq 0.2$. $\tau_{F 0}$ is still a

\begin{tabular}{lcccc}
\hline Mesh & Nb of elem. & $h / \tau_{F}$ & $\tau_{F 0} / \tau_{F}$ & $L_{F 0} / \widetilde{L}$ \\
\hline 1 (uniform) & 230 & 0.25 & 0.905 & 2.55 \\
2 (uniform) & 1894 & 0.25 & 0.968 & 2.64 \\
3 (uniform, ref.) & $1.2410^{6}$ & 0.25 & 0.991 & 2.67 \\
4 (not uniform) & 2123 & 0.25 & 0.935 & 2.59 \\
\hline 4 (not uniform) & 2123 & $1 / 75$ & - & - \\
5 (not uniform, ref.) & $0.29510^{6}$ & $1 / 1500$ & - & - \\
\hline
\end{tabular}

Table 1: Discretized problem characteristics and obtained exponential decay. 


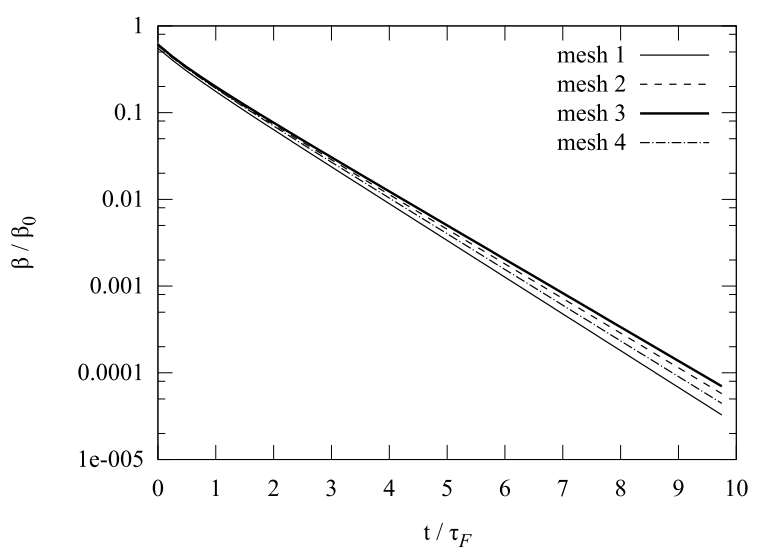

Figure 6: Obtained evolutions of the memory function at large time, using coarse time steps. Influence of the spatial discretization.
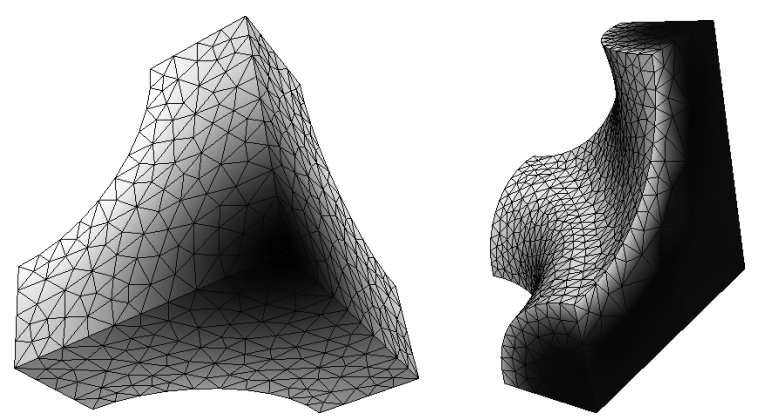

Figure 7: Left: mode for the long term temperature evolution, as computed on mesh 2. Right: transient solution at $t / \tau_{F}=2.6710^{-2}$, as computed on mesh 4 .

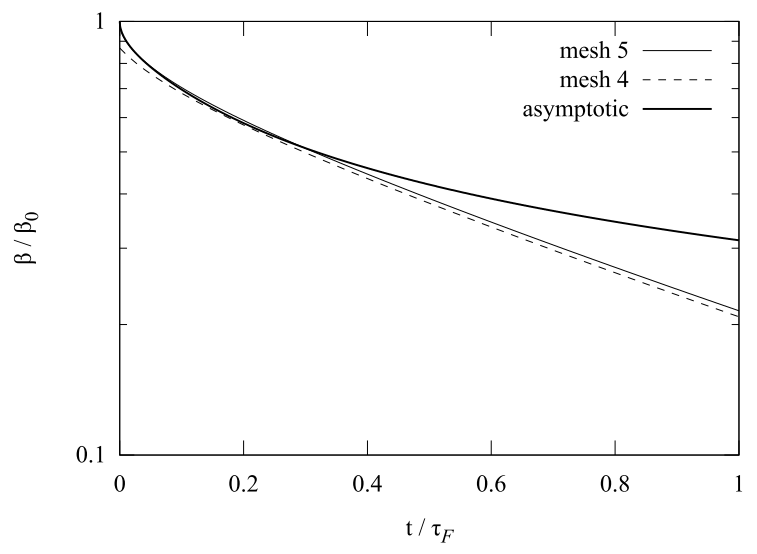

Figure 8: Obtained evolutions of the memory function at small time. Influence of the spatial discretization and comparison with the approximation for small time values. 
quantity to estimate, or to evaluate using the previous iterative power method on a uniform mesh an a coarse time step. Once obtained, it can be used to determine its associated characteristic length $L_{F 0}=\pi \sqrt{\tau_{F 0} \rho_{F} c_{F} / k_{F}^{\prime}}$; then one may choose $\tau_{F}=\tau_{F 0}$ and $L_{F}=L_{F 0}$, which, together with the previously proposed estimation of $\widetilde{L}$ allows to reconstruct the asymptotic solution.

\section{Applications}

The applications of the proposed numerical modeling approach participate to the material design theme. Indeed, memory effects may be viewed as either a goal for design of functional materials (indeed, closedform expressions also allows optimization for goal-oriented design microstructures), or as a drawback that have to be checked for structural integrity.

For instance, thermal delayed effects obtained by highly heterogeneous materials can be a simpler alternative than those obtained by phase changes for applications in building thermal efficiency [25, 26]. If the characteristic time of memory function could be of the order of the day, the contrast in day and night temperatures could be counterbalanced.

Other diffusion-involved physics are also under concern: for instance the porous media flows [9] that may exhibit such delayed effects at several scales, for double permeability media, to be taken into account for contaminant dispersion in soils, or for oil recovery [27], the blood perfusion in biological tissues or the compact bone porous media [28].

For the case of multifunctional designs, some microstructured composite materials may be used for other purpose-oriented applications, leading to material phase selections that exhibit a large contrast in thermal properties [29]. The thermomechanical behavior of these materials have to be checked and memory effects have to be taken into account for application with thermal transient loadings. Indeed, analyses for a phenomenological model (without memory effect) and an homogenized one may lead to quantitative different responses [30].

\section{Conclusions}

This article proposes a systematic numerical approach to derive the macroscopic memory effect function for composite material with two phases and a large contrast in their thermal properties. Using the periodic homogenization procedure, it derives the associated microscopic transient problem on a representative cell. The proposed numerical algorithm is related to the spectral properties of the incremental operator traducing the thermal transient behavior of the low thermal conduction phase.

Approximate simple forms of the memory function are provided, one for the short time memory effect, and one for the vanishing time effect. They do not require any 3D simulation, but they condense the microstructure complexity in two scalar parameters, namely a characteristic length and a characteristic time. Though estimates of these two parameters are provided, their validity on a large range of various complex microstructures is still to be validated. This validation is nevertheless exemplified in this article for two different cases: a simple 1D problem for which an exact analytical solution is available, and a 3D problem for which a $3 \mathrm{D}$ numerical simulation is performed.

\section{References}

[1] J. Lemaitre, J.-L. Chaboche, Mechanics of Solid Materials, Cambridge University Press, 1994.

[2] G. Allaire, Homogenization and two-scale convergence, SIAM Journal on Mathematical Analysis 23 (6) (1992) 1482-1518.

[3] J. Fish, W. Chen, Uniformly valid multiple spatial-temporal scale modeling for wave propagation in heterogeneous media, Mechanics of Composite Materials and Structures 8 (2001) 81-99.

[4] P. Suquet, Effective properties of time dependent heterogeneous materials, in: 9th International Conference on the Mechanics of Time Dependent Materials, Montreal, Canada, 2014.

[5] Z. Abdessamad, I. Kostin, G. Panasenko, V. P. Smyshlyaev, Memory effect in homogenization of a viscoelastic Kelvin-Voigt model with time-dependent coefficients, Mathematical Models and Methods in Applied Sciences 19 (09) (2009) 1603-1630. doi:10.1142/S0218202509003905. 
[6] J.-S. Jiang, Two-scale homogenization and memory effects of a first order differential equation, Taiwanese Journal of Mathematics 10 (4) (2006) 963-976.

[7] J.-L. Auriault, Effective macroscopic description for heat conduction in periodic composites, International Journal of Heat and Mass Transfer 26 (6) (1983) 861- 869. doi:10.1016/S0017-9310(83)801100 .

[8] L. Tartar, Memory effects and homogenization, Archive for Rational Mechanics and Analysis 111 (2) (1990) 121-133. doi:10.1007/BF00375404.

[9] P. Royer, C. Boutin, Time analysis of the three characteristic behaviours of dual-porosity media. I: Fluid flow and solute transport, Transport in Porous Media 95 (3) (2012) 603-626. doi:10.1007/s11242-012-0065-2.

[10] J.-L. Auriault, Upscaling heterogeneous media by asymptotic expansions, Journal of Engineering Mechanics 128 (8) (2002) 817-822. doi:10.1061/(ASCE)0733-9399(2002)128:8(817).

[11] E. Sanchez-Palencia, Non homogeneous media and vibration theory, Vol. 127 of Lecture Notes in Physics, Springer Verlag, 1980.

[12] A. Bensoussan, J.-L. Lions, G. Papanicolaou, Asymptotic Analysis for Periodic Structures, North Holland, Amsterdam, 1978.

[13] D. Néron, D. Dureisseix, A computational strategy for thermo-poroelastic structures with a timespace interface coupling, International Journal for Numerical Methods in Engineering 75 (9) (2008) 1053-1084. doi:10.1002/nme.2283.

[14] F. Feyel, J.-L. Chaboche, $\mathrm{FE}^{2}$ multiscale approach for modelling the elastoviscoplastic behaviour of long fiber SiC/Ti composite materials, Computer Methods in Applied Mechanics and Engineering 183 (2000) 309-330. doi:10.1016/S0045-7825(99)00224-8.

[15] P. Ciarlet, P.-A. Raviart, Maximum principle and uniform convergence for the finite element method, Computer Methods in Applied Mechanics and Engineering 2 (1) (1973) 17 - 31. doi:10.1016/00457825(73)90019-4.

[16] V. Thomée, L. B. Wahlbin, On the existence of maximum principles in parabolic finite element equations, Mathematics of Computation 77 (261) (2008) 1119, s 0025-5718(07)02021-2.

[17] Hibbitt, Karlson, Sorensen (Eds.), Abaqus/Standard — User's Manual, Vol. I, 1996, pp. 6.4.2-2 and 6.6.1-4.

[18] P. A. Vermeer, A. Veruijt, An accuracy condition for consolidation by finite elements, International Journal for Numerical and Analytical Methods in Geomechanics 5 (1) (1981) 1-14. doi:10.1002/nag.1610050103.

[19] H. Fujii, Some remarks on finite element analysis of time-dependent field problems, Theory and practice in finite element structural analysis (1973) 91-106.

[20] A. El-Zein, Exponential finite elements for diffusion-advection problems, International Journal for Numerical Methods in Engineering 62 (2005) 2086-2103. doi:10.1002/nme.1249.

[21] D. Turner, D. Noble, Analytic enrichment of finite element formulations for capturing boundary layer behavior, in: 40th Fluid Dynamics Conference and Exhibit, American Institute of Aeronautics and Astronautics, 2010, pp. 2675-2693. doi:10.2514/6.2010-4996.

[22] C. Geuzaine, J.-F. Remacle, Gmsh: A 3-D finite element mesh generator with built-in pre- and post-processing facilities, International Journal for Numerical Methods in Engineering 79 (2009) 1309-1331. doi:10.1002/nme.2579.

[23] A. Henderson, J. Ahrens, C. Law, The ParaView Guide, Kitware Inc., Clifton Park, NY, 2004.

[24] Cast3M web site, http://www-cast3m.cea.fr, accessed: 2015-01-09.

[25] A. Sharma, V. V. Tyagi, C. R. Chen, D. Buddhi, Review on thermal energy storage with phase change materials and applications, Renewable and Sustainable Energy Reviews 13 (9) (2009) 318345. doi:10.1016/j.rser.2007.10.005. 
[26] F. Kuznik, D. David, K. Johannes, J.-J. Roux, A review on phase change materials integrated in building walls, Renewable and Sustainable Energy Reviews 15 (2011) 379-391. doi:10.1016/j.rser.2010.08.019.

[27] M. Enamul Hossain, L. Lie, M. Rafiqul Islam, Inclusion of the memory function in describing the flow of shear-thinning fluids in porous media, International Journal of Engineering 3 (5) (2009) $458-478$.

[28] E. Rohan, R. Cimrman, Multiscale FE simulation of diffusion-deformation processes in homogenized dual-porous media, Mathematics and Computers in Simulation 82 (10) (2012) 1744-1772. doi:10.1016/j.matcom.2011.02.011.

[29] D. L. Ellis, D. L. McDanels, Thermal conductivity and thermal expansion of graphite fiber/copper matrix composites, Technical Memorandum TM-105233, NASA Lewis Research Center, Cleveland, Ohio (1991).

[30] J. L. Auriault, P. Royer, Double conductivity media: a comparison between phenomenological and homogenization approaches, International Journal of Heat and Mass Transfer 36 (10) (1993) 26132621. doi:10.1016/S0017-9310(05)80198-X. 